\title{
STATE MAPS FROM INTEGRATION BY PARTS*
}

\author{
ARJAN VAN DER SCHAFT ${ }^{\dagger}$ AND PAOLO RAPISARDA A $^{\ddagger}$
}

\begin{abstract}
We develop a new approach to the construction of state vectors for linear timeinvariant systems described by higher-order differential equations. The basic observation is that the concatenation of two solutions of higher-order differential equations results in another (weak) solution once their remainder terms resulting from (repeated) integration by parts match. These remainder terms can be computed in a simple and efficient manner by making use of the calculus of bilinear differential forms and two-variable polynomial matrices. Factorization of the resulting two-variable polynomial matrix defines a state map, as well as a state map for the adjoint system. Minimality of these state maps is characterized. The theory is applied to three classes of systems with additional structure, namely self-adjoint Hamiltonian, conservative port-Hamiltonian, and timereversible systems. For the first two classes it is shown how the factorization leading to a (minimal) state map is equivalent to the factorization of another two-variable polynomial matrix, which is immediately derived from the external system characterization, and defines a symplectic, respectively, symmetric, bilinear form on the minimal state space.
\end{abstract}

Key words. adjoint systems, Hamiltonian systems, state, integration by parts, factorization

AMS subject classifications. 93B20, 34A30, 37J99

DOI. $10.1137 / 100806825$

1. Introduction. The notion of state is central in systems and control theory. Control strategies such as optimal control are based on the current relevant information about the system captured by its state, and state space models often turn out to be analytically and computationally most advantageous. Thus questions arise of how to construct a (minimal) state and how to "realize" an input-output behavior by a set of first-order differential equations in the, yet to be defined, state variables. Furthermore, there is a clear interest per se in understanding the relation between system properties at the external - input and output - level, and at the internal - state - level.

In this paper we take a fresh look at the problem of determining a (minimal) state for systems described by linear higher-order differential equations,

$$
P\left(\frac{d}{d t}\right) y(t)=Q\left(\frac{d}{d t}\right) u(t), \quad y(t) \in \mathcal{Y}:=\mathbb{R}^{p}, u(t) \in \mathcal{U}:=\mathbb{R}^{m},
$$

or more generally, without distinguishing between inputs $u$ and outputs $y$ and letting $w:=\left[\begin{array}{l}y \\ u\end{array}\right], q:=p+m$,

$$
R\left(\frac{d}{d t}\right) w(t)=0, \quad w(t) \in \mathcal{W}:=\mathbb{R}^{q} .
$$

* Received by the editors August 27, 2010; accepted for publication (in revised form) September 9, 2011; published electronically November 17, 2011. A preliminary version of some of the results in this paper has been presented in [15].

http://www.siam.org/journals/sicon/49-6/80682.html

$\dagger$ Johann Bernoulli Institute for Mathematics and Computer Science, University of Groningen, P.O. Box 407, 9700 AK Groningen, The Netherlands (a.j.van.der.schaft@rug.nl).

${ }^{\ddagger}$ ISIS Group, School of Electronics and Computer Science, University of Southampton, SO17 1BJ Southampton, United Kingdom (pr3@ecs.soton.ac.uk). The research of this author was done during a sabbatical period supported financially by grant EP/I000909/1 of the United Kingdom Engineering and Physical Sciences Research Council. 
In all of these equations, $P\left(\frac{d}{d t}\right), Q\left(\frac{d}{d t}\right)$, and $R\left(\frac{d}{d t}\right)$ describe linear (higher-order) differential operators, or, equivalently, $P(\xi), Q(\xi)$, and $R(\xi)$ are polynomial matrices of appropriate dimensions in the indeterminate $\xi$.

It is well known $[11,14]$ that for an observable input-state-output system

$$
\begin{aligned}
\frac{d}{d t} x & =A x+B u, \quad x(t) \in \mathbb{R}^{n}, u(t) \in \mathbb{R}^{m} \\
y & =C x+D u, \quad y(t) \in \mathbb{R}^{p}
\end{aligned}
$$

the state $x$ can be written as a linear combination of the outputs and inputs and their derivatives, i.e., $x=X_{y}\left(\frac{d}{d t}\right) y+X_{u}\left(\frac{d}{d t}\right) u$ for certain linear differential operators $X_{y}\left(\frac{d}{d t}\right), X_{u}\left(\frac{d}{d t}\right)$, or, more compactly,

$$
x=X\left(\frac{d}{d t}\right) w
$$

for some $n \times q$ polynomial matrix $X(\xi)$. We will call (1.4) a state map.

The first, and most basic, question we want to address in this paper is the possibility of a "canonical" construction of a state map based on the higher-order description (1.1) or (1.2). Indeed, while there are various methods for coming up with state space representations (1.3) for system (1.1) or (1.2), they are mostly algorithmically oriented and do not provide an intrinsic link between the differential operator $X\left(\frac{d}{d t}\right)$ (or $X_{y}\left(\frac{d}{d t}\right)$ and $\left.X_{u}\left(\frac{d}{d t}\right)\right)$ describing the state map and the differential operator $R\left(\frac{d}{d t}\right)$ (respectively, $P\left(\frac{d}{d t}\right)$ and $Q\left(\frac{d}{d t}\right)$ ) describing the original higher-order linear system. In this paper we will show that, indeed, such a canonical construction can be given, and it is reliant upon a very classical notion, namely integration by parts. In fact, it will be shown in section 2 that state maps can be constructed by factorization of a two-variable polynomial matrix corresponding to the remainders in performing repeated integration by parts to (1.1) or (1.2).

Besides its intrinsic value, this new approach to constructing state maps offers a number of important advantages. First of all, because the state map is directly based on the differential operator $R\left(\frac{d}{d t}\right)$ describing the external (input-output) behavior of the system, it is easy to translate properties of the external behavior of the system into its internal (state) behavior. This will be illustrated in section 4, where it will be shown how two versions of external Hamiltonian behavior, namely self-adjoint Hamiltonian systems and conservative port-Hamiltonian systems, directly translate into internal behavior. In particular, we will show how the external behavior in both cases explicitly defines an internal structure on the state space defined by the canonical state map construction developed in section 2. In the first case (self-adjoint Hamiltonian systems) this is the symplectic structure on this state space, while in the second case (conservative port-Hamiltonian systems) this is the explicitly constructed internal energy function. We also show how the property of time-reversibility of the external behavior immediately translates into the explicit construction of an involution on the canonically defined state. In [25] we have applied the same methodology to the relation between external and internal system decompositions. Compared to the existing literature on these and related subjects, a main novelty of the approach taken in this paper is that for the derivation of, e.g., the symplectic structure or internal energy we do not rely on the state space isomorphism theorem for minimal systems (guaranteeing the existence of such objects), but instead we explicitly construct such objects on the canonically defined state space. 
Surprisingly enough, our canonical state map construction also yields a canonical state map for the adjoint system. In fact, while one of the two terms in the factorization of the two-variable polynomial matrix corresponding to the remainders yields the state map for the original system, the other defines a state map for the adjoint system. These connections and their consequences (e.g., for uncontrollable systems) are explored in section 3 .

Another advantage of our approach, to be explored in future work, is its potential for generalizations to systems described by (higher-order) linear PDEs, or even to nonlinear systems. Indeed, because our approach is based on the fundamental notion of integration by parts, it is in principle extendable to much more general situations than linear time-invariant ODEs.

Finally, we remark that the canonical state map construction developed in this paper also yields a new, and transparent, algorithm to effectively compute state maps and eventually state space realizations. The consequences of this algorithm for state space realization theory will be detailed in a future paper.

1.1. Mathematical background and notation. The main mathematical tools employed in this paper concern the correspondence between linear differential operators and polynomial matrices, and that between bilinear differential forms (scalar-valued bilinear differential operators) and two-variable polynomial matrices. For additional background concerning the former correspondence, see [14]; about the latter correspondence, we refer the reader to [27].

Throughout the paper, $\mathbb{R}^{p \times q}[\xi]$ will denote the space of real $p \times q$ polynomial matrices in the indeterminate $\xi$, while $\mathbb{R}^{p \times q}[\zeta, \eta]$ will denote the space of real $p \times q$ polynomial matrices in the indeterminates $\zeta$ and $\eta$. $R^{T}(\xi)$, respectively, $\Phi^{T}(\zeta, \eta)$, will denote the transpose of $R(\xi) \in \mathbb{R}^{p \times q}[\xi]$, respectively, of $\Phi(\zeta, \eta) \in \mathbb{R}^{p \times q}[\zeta, \eta]$.

\section{State maps from integration by parts.}

2.1. The notion of state. Consider the system of linear higher-order differential equations

$$
R\left(\frac{d}{d t}\right) w(t)=0, \quad w(t) \in \mathcal{W}:=\mathbb{R}^{q},
$$

where $R(\xi)=R_{0}+R_{1} \xi^{1}+\cdots+R_{N} \xi^{N} \in \mathbb{R}^{p \times q}[\xi]$. Denote the space of locally integrable trajectories from $\mathbb{R}$ to $\mathbb{R}^{q}$ by $\mathcal{L}_{1}^{\text {loc }}\left(\mathbb{R}, \mathbb{R}^{q}\right)$. Recall that $w \in \mathcal{L}_{1}^{\text {loc }}\left(\mathbb{R}, \mathbb{R}^{q}\right)$ is a weak solution of (2.1) if

$$
\int_{-\infty}^{\infty} w^{T}(t) R^{T}\left(-\frac{d}{d t}\right) \varphi(t) d t=0
$$

for all $\mathcal{C}^{\infty}$ test functions $\varphi: \mathbb{R} \rightarrow \mathbb{R}^{p}$ with compact support. The set of weak solutions of $(2.1)$, also called the behavior $\mathcal{B}$, is defined as (see, e.g., $[10,14,17]$ )

$$
\mathcal{B}:=\left\{w: \mathbb{R} \rightarrow \mathbb{R}^{q} \mid w \in \mathcal{L}_{1}^{\text {loc }}\left(\mathbb{R}, \mathbb{R}^{q}\right) \text { and (2.1) is satisfied weakly }\right\} .
$$

In behavioral system theory (see, e.g., $[14,17]$ ), the following transparent definition of state has been developed. Consider two solutions $w_{1}, w_{2} \in \mathcal{B}$, and define the concatenation of $w_{1}$ and $w_{2}$ at time 0 as the time-function

$$
\left(w_{1} \wedge_{0} w_{2}\right)(t):=\left\{\begin{array}{ll}
w_{1}(t), & t<0, \\
w_{2}(t), & t \geq 0,
\end{array} \quad t \in \mathbb{R} .\right.
$$


We say that $w_{1}, w_{2} \in \mathcal{B}$ are equivalent at time 0 , denoted as $w_{1} \sim_{0} w_{2}$, if for all $w \in \mathcal{B}$,

$$
w_{1} \wedge_{0} w \in \mathcal{B} \Leftrightarrow w_{2} \wedge_{0} w \in \mathcal{B} .
$$

Thus equivalent trajectories admit the same continuations starting from time $t=0$.

Remark 2.1. In the context of linear systems (as in this paper), this is equivalent to requiring that $w_{1} \wedge_{0} w$ and $w_{2} \wedge_{0} w \in \mathcal{B}$ for some $w \in \mathcal{B}$. Furthermore, in this case, since $w_{2} \wedge_{0} w_{2} \in \mathcal{B}$, it follows that $w_{1} \sim_{0} w_{2}$ if and only $w_{1} \wedge_{0} w_{2} \in \mathcal{B}$. Because of the symmetry of this last condition, it also means that equivalence of $w_{1}, w_{2} \in \mathcal{B}$ at $t=0$ amounts to $w_{1}$ and $w_{2}$ having the same precursors. (Note that for nonlinear systems these equivalences in general do not hold; see [22] for some initial ideas about the construction of state maps in this case.)

Let $X(\xi) \in \mathbb{R}^{n \times q}[\xi]$. Then the differential operator

$$
\begin{aligned}
X\left(\frac{d}{d t}\right): \mathcal{L}_{1}^{\text {loc }}\left(\mathbb{R}, \mathbb{R}^{q}\right) & \rightarrow \mathcal{L}_{1}^{\text {loc }}\left(\mathbb{R}, \mathbb{R}^{n}\right), \\
w & \mapsto x:=X\left(\frac{d}{d t}\right) w
\end{aligned}
$$

is said to be a state map [17] for the system (2.1), with set of solutions $\mathcal{B}$ defined in (2.3), if for all $w_{1}, w_{2} \in \mathcal{B}$ and corresponding $x_{i}:=X\left(\frac{d}{d t}\right) w_{i}, i=1,2$, the following property (the state property) holds:

$$
\left[x_{1}(0)=x_{2}(0)\right] \text { and }\left[x_{1}, x_{2} \text { continuous at } t=0\right] \Longrightarrow\left[w_{1} \sim_{0} w_{2}\right] .
$$

If (2.6) holds, then the vector $x$ contains all the information necessary to conclude whether any two trajectories in $\mathcal{B}$ admit the same continuation at time $t=0$. For this reason the vector $x(0)=X\left(\frac{d}{d t}\right) w(0)$ is called a state of the system at ${ }^{1}$ time 0 corresponding to the time-function $w$, and $\mathcal{X}=\mathbb{R}^{n}$ is called a state space for the system. If $n$ is minimal among all the state vector dimensions, then the state map is called a minimal state map. We then call $n$ the McMillan degree of the system.

2.2. Integration by parts and bilinear differential forms. Our starting point for obtaining state maps is the basic integration by parts formula. Take any $N$ times differentiable functions $w: \mathbb{R} \rightarrow \mathbb{R}^{q}$ and $\varphi: \mathbb{R} \rightarrow \mathbb{R}^{p}$ and denote $w^{(i)}:=\frac{d^{i}}{d t^{i}} w$, $\varphi^{(i)}:=\frac{d^{i}}{d t^{2}} \varphi, i \in \mathbb{N}$. For each pair of time instants $t_{1} \leq t_{2}$, repeated integration by parts yields

$$
\int_{t_{1}}^{t_{2}} w^{T}(t) R^{T}\left(-\frac{d}{d t}\right) \varphi(t) d t=\int_{t_{1}}^{t_{2}} \varphi^{T}(t) R\left(\frac{d}{d t}\right) w(t) d t+\left.B_{\Pi}(\varphi, w)\right|_{t_{1}} ^{t_{2}}
$$

where we call the expression $B_{\Pi}(\varphi, w)(t)$ the remainder, which has the form

$$
B_{\Pi}(\varphi, w)(t)=\left[\begin{array}{llll}
\varphi^{T}(t) & \varphi^{(1) T}(t) & \cdots & \varphi^{(N-1) T}(t)
\end{array}\right] \tilde{\Pi}\left[\begin{array}{c}
w(t) \\
w^{(1)}(t) \\
\vdots \\
w^{(N-1)}(t)
\end{array}\right],
$$

for some constant matrix $\tilde{\Pi}$ of dimension $N p \times N q$.

\footnotetext{
${ }^{1}$ Of course, by time-invariance, $x(t)=X\left(\frac{d}{d t}\right) w(t)$ defines a state of the system at any time $t$.
} 
The differential version of the integration by parts formula (2.7) (obtained by dividing (2.7) by $t_{2}-t_{1}$ and letting $t_{1}$ tend to $t_{2}=t$ ) is

$$
w^{T}(t) R^{T}\left(-\frac{d}{d t}\right) \varphi(t)-\varphi^{T}(t) R\left(\frac{d}{d t}\right) w(t)=\frac{d}{d t} B_{\Pi}(\varphi, w)(t) .
$$

Both sides of this equality define a bilinear differential operator form or, briefly, $b i$ linear differential form (BDF), i.e., a bilinear functional of two trajectories and of a finite number of their derivatives. Formally, a BDF $B_{\Phi}$ as defined in [27] is a bilinear map $B_{\Phi}: \mathcal{C}^{\infty}\left(\mathbb{R}, \mathbb{R}^{p}\right) \times \mathcal{C}^{\infty}\left(\mathbb{R}, \mathbb{R}^{q}\right) \rightarrow \mathcal{C}^{\infty}(\mathbb{R}, \mathbb{R})$ involving two vector-valued functions and a finite set of their time-derivatives, that is, at any time $t$,

$$
B_{\Phi}(\varphi, w)(t)=\sum_{k, l=0}^{M-1}\left[\frac{d^{k}}{d t^{k}} \varphi(t)\right]^{T} \Phi_{k, l} \frac{d^{l}}{d t^{l}} w(t)
$$

for certain constant $p \times q$ matrices $\Phi_{k, l}, k, l=0, \ldots, M-1$. The matrix $\tilde{\Phi}$ whose $(k, l)$ th block is the matrix $\Phi_{k, l}$ for $k, l=0, \ldots, M-1$, is called the coefficient matrix of the bilinear differential form $B_{\Phi}$. It follows that the coefficient matrix of the bilinear differential form $B_{\Pi}$ corresponding to the remainder is precisely the matrix $\tilde{\Pi}$ as defined in (2.8).

Remark 2.2. For a scalar polynomial or a square polynomial matrix $R(\xi)$ the formulas (2.7) and (2.9) are classically referred to as Green's, respectively, Lagrange's, identity, while the matrix $\tilde{\Pi}$ for a scalar $R(\xi)$ is called the bilinear concomitant; see [10].

There is a useful one-to-one correspondence between the bilinear differential form $B_{\Phi}$ in (2.10) and the two-variable polynomial matrix $\Phi(\zeta, \eta)$ defined as

$$
\Phi(\zeta, \eta):=\sum_{k, l=0}^{M-1} \Phi_{k, l} \zeta^{k} \eta^{l} .
$$

The crucial observation (see $[3,27]$ ) is that for any bilinear differential form $B_{\Phi}$ the bilinear differential form corresponding to its time-derivative, defined as

$$
\begin{aligned}
B_{\Psi} & (\varphi, w)(t):=\frac{d}{d t}\left(B_{\Phi}(\varphi, w)\right)(t) \\
= & \sum_{k, l=0}^{M-1}\left[\frac{d^{k+1}}{d t^{k+1}} \varphi(t)\right]^{T} \Phi_{k, l} \frac{d^{l}}{d t^{l}} w(t)+\left[\frac{d^{k}}{d t^{k}} \varphi(t)\right]^{T} \Phi_{k, l} \frac{d^{l+1}}{d t^{l+1}} w(t),
\end{aligned}
$$

corresponds, by the product rule of differentiation, to the two-variable polynomial matrix

$$
\Psi(\zeta, \eta)=(\zeta+\eta) \Phi(\zeta, \eta)
$$

As a consequence, the differential version of the integration by parts formula (2.9) has associated with it the two-variable polynomial matrix equality

$$
R(-\zeta)-R(\eta)=(\zeta+\eta) \Pi(\zeta, \eta) .
$$

From this formula it follows how the two-variable polynomial matrix $\Pi(\zeta, \eta)$ and its coefficient matrix $\tilde{\Pi}$ (corresponding to the remainder) can be most easily computed: 
since the two-variable polynomial matrix $R(-\zeta)-R(\eta)$ is zero for $\zeta+\eta=0$, it directly follows that $R(-\zeta)-R(\eta)$ contains a factor $\zeta+\eta$, and thus we can define the two-variable polynomial matrix $\Pi(\zeta, \eta)$ as

$$
\Pi(\zeta, \eta):=\frac{R(-\zeta)-R(\eta)}{\zeta+\eta} .
$$

2.3. State maps from factorization. It turns out that state maps for a system $R\left(\frac{d}{d t}\right) w=0$ can be computed from a factorization of the two-variable polynomial matrix $\Pi(\zeta, \eta)$ into a product of single-variable polynomial matrices. Before showing this, we recall how factorizations of the coefficient matrix of a bilinear differential form correspond to factorizations of the two-variable polynomial matrix corresponding to it. This is summarized in the following proposition.

Proposition 2.3. Let $\Phi \in \mathbb{R}^{p \times q}[\zeta, \eta]$, and let $\tilde{\Phi}$ be its coefficient matrix. Then the following two statements are equivalent:

1. There exist real matrices $\tilde{F}, \tilde{G}$ with $n$ rows such that

$$
\tilde{\Phi}=\tilde{F}^{T} \tilde{G} .
$$

2. There exist polynomial matrices $F \in \mathbb{R}^{n \times p}[\xi], G \in \mathbb{R}^{n \times q}[\xi]$ with coefficient matrices $\tilde{F}, \tilde{G}$, i.e.,

$$
F(\xi)=\tilde{F}\left[\begin{array}{c}
I_{p} \\
\xi I_{p} \\
\vdots
\end{array}\right] \text { and } G(\xi)=\tilde{G}\left[\begin{array}{c}
I_{q} \\
\xi I_{q} \\
\vdots
\end{array}\right],
$$

such that

$$
\Phi(\zeta, \eta)=F^{T}(\zeta) G(\eta) .
$$

Proof. This follows from the discussion on page 1709 of [27].

Factorizations such as those of Proposition 2.3, which, moreover, correspond to the minimal value $n=\operatorname{rank}(\tilde{\Phi})$, are called minimal (or canonical as in [27]). Note that the matrices $\tilde{F}$ and $\tilde{G}$ involved in a minimal factorization of $\tilde{\Phi}$ are of full row rank.

Minimal factorizations are not unique; however, using standard linear algebra arguments, the following proposition can be easily verified.

Proposition 2.4. Given a minimal factorization $\tilde{\Phi}=\tilde{F}^{T} \tilde{G}$, every other minimal factorization $\tilde{\Phi}=\tilde{F}^{\prime T} \tilde{G}^{\prime}$ can be obtained from it by premultiplication of $\tilde{F}$ and $\tilde{G}$ by a nonsingular $n \times n$ matrix $S$, respectively, $S^{-T}$. In view of Proposition 2.3 this implies that $\Phi(\zeta, \eta)=F^{T}(\zeta) G(\eta)=F^{\prime T}(\zeta) G^{\prime}(\eta)$ with $F^{\prime}(\xi):=S F(\xi), G^{\prime}(\xi):=S^{-T} G(\xi)$.

Any factorization $\Pi(\zeta, \eta)=Y^{T}(\zeta) X(\eta)$ of the two-variable polynomial matrix $\Pi(\zeta, \eta)$ corresponding to the remainder leads from (2.14) to the matrix polynomial equality

$$
R(-\zeta)-R(\eta)=(\zeta+\eta) Y^{T}(\zeta) X(\eta)
$$

and to the corresponding bilinear differential form equality expanding (2.9),

$$
\begin{aligned}
w^{T}(t) R^{T}\left(-\frac{d}{d t}\right) \varphi(t) & -\varphi^{T}(t) R\left(\frac{d}{d t}\right) w(t) \\
& =\frac{d}{d t}\left[\left(Y\left(\frac{d}{d t}\right) \varphi(t)\right)^{T} X\left(\frac{d}{d t}\right) w(t)\right],
\end{aligned}
$$

Copyright (c) by SIAM. Unauthorized reproduction of this article is prohibited. 
which immediately leads to the construction of a state map, as the following result states.

Theorem 2.5. For any factorization $\Pi(\zeta, \eta)=Y^{T}(\zeta) X(\eta)$, the map

$$
w \mapsto x:=X\left(\frac{d}{d t}\right) w
$$

is a state map.

Proof. We begin by proving the following result.

Lemma 2.6. Let $\Pi(\zeta, \eta)=Y^{T}(\zeta) X(\eta)$ be a factorization. Then for any $w_{1}, w_{2} \in$ $\mathcal{B}$ it holds that

$$
\left[X\left(\frac{d}{d t}\right) w_{1}(0)=X\left(\frac{d}{d t}\right) w_{2}(0)\right] \Longrightarrow\left[w_{1} \sim_{0} w_{2}\right] .
$$

Furthermore, if $\Pi(\zeta, \eta)=Y^{T}(\zeta) X(\eta)$ is a minimal factorization, then also the converse implication of (2.18) holds.

Proof. We first consider $\mathcal{C}^{\infty}$ trajectories $w_{1}, w_{2}, w \in \mathcal{B}$. By definition, $w_{1} \wedge_{0} w \in \mathcal{B}$ if and only if

$$
\int_{-\infty}^{\infty}\left(w_{1} \wedge w\right)^{T}(t) R^{T}\left(-\frac{d}{d t}\right) \varphi(t) d t=0
$$

for all $\mathcal{C}^{\infty}$ test functions $\varphi$ with compact support; an analogous expression holds for $w_{2}$. Integrating by parts on the two intervals $(-\infty, 0)$ and $(0, \infty)$ and using $R\left(\frac{d}{d t}\right) w_{1}(t)=0$ for $t<0$ and $R\left(\frac{d}{d t}\right) w_{2}(t)=0$ for $t \geq 0$ yields that this holds if and only if

$$
\left(Y\left(\frac{d}{d t}\right) \varphi(0)\right)^{T} X\left(\frac{d}{d t}\right) w_{1}(0)=\left(Y\left(\frac{d}{d t}\right) \varphi(0)\right)^{T} X\left(\frac{d}{d t}\right) w(0)
$$

for all $\mathcal{C}^{\infty}$ test functions $\varphi$ with compact support. The same holds for the concatenation of $w_{2}$ and $w$. Thus $w_{1} \sim_{0} w_{2}$ if and only if

$$
\left(Y\left(\frac{d}{d t}\right) \varphi(0)\right)^{T} X\left(\frac{d}{d t}\right) w_{1}(0)=\left(Y\left(\frac{d}{d t}\right) \varphi(0)\right)^{T} X\left(\frac{d}{d t}\right) w_{2}(0)
$$

for all $\varphi$.

Hence $X\left(\frac{d}{d t}\right) w_{1}(0)=X\left(\frac{d}{d t}\right) w_{2}(0)$ implies $w_{1} \sim_{0} w_{2}$, thus proving (2.18). Furthermore, in the case of $\Pi(\zeta, \eta)=Y^{T}(\zeta) X(\eta)$ is a minimal factorization, then given the arbitrariness of $\varphi$ and the injectivity of $\tilde{Y}^{T}$, it follows that if $w_{i} \in \mathcal{B}, i=1,2$, and (2.19) holds, then also $X\left(\frac{d}{d t}\right) w_{1}(0)=X\left(\frac{d}{d t}\right) w_{2}(0)$, and consequently the converse implication of (2.18) holds. Since the subset of $\mathcal{C}^{\infty}$ trajectories in $\mathcal{B}$ is dense in $\mathcal{B}$ (in the sense of $\mathcal{L}_{1}^{\text {loc }}\left(\mathbb{R}, \mathbb{R}^{q}\right)$ ) (cf. Corollary 2.4.12 in [14]), the result of the lemma follows.

To complete the proof of Theorem 2.5, observe that the differential operator $X\left(\frac{d}{d t}\right)$ obtained from any factorization of $\Pi$ is such that for all $w \in \mathcal{B}$ the trajectory $X\left(\frac{d}{d t}\right) w$ is continuous, since $X\left(\frac{d}{d t}\right) w$ is a linear combination of expressions obtained by integrating by parts an $\mathcal{L}_{1}^{\text {loc }}\left(\mathbb{R}, \mathbb{R}^{q}\right)$ function, and is consequently an absolutely continuous time-function; see page 1062 of [17] for details. 
Remark 2.7. It is a matter of straightforward verification to check that the coefficient matrix $\tilde{\Pi}$ of $\Pi(\zeta, \eta)$ equals

$$
\tilde{\Pi}=\left[\begin{array}{ccccc}
-R_{1} & -R_{2} & \cdots & -R_{N-1} & -R_{N} \\
R_{2} & R_{3} & \cdots & R_{N} & 0 \\
\vdots & \vdots & . \cdot & \vdots & \vdots \\
(-1)^{N-1} R_{N-1} & (-1)^{N-1} R_{N} & 0 & \cdots & 0 \\
(-1)^{N} R_{N} & 0 & 0 & \cdots & 0
\end{array}\right]
$$

(which in the scalar case is classically known as the bilinear concomitant [10]). Up to minus signs, this corresponds to the expression obtained by applying the shift-andcut map on the matrix $R(\xi)$ (see section 5 of [17]), which we now briefly recall. Any rational function $q(\xi) \in \mathbb{R}(\xi)$ can be uniquely written as the sum of a polynomial $p_{q}(\xi)$ and of a strictly proper rational function $s_{q}(\xi)$. Now define

$$
\begin{aligned}
()_{+}: \mathbb{R}(\xi) & \rightarrow \mathbb{R}[\xi], \\
q(\xi) & \mapsto(q(\xi))_{+}:=p_{q}(\xi) .
\end{aligned}
$$

The shift-and-cut map $\sigma_{+}: \mathbb{R}[\xi] \rightarrow \mathbb{R}[\xi]$ is defined as

$$
\sigma_{+}(p(\xi)):=\left(\xi^{-1} p(\xi)\right)_{+},
$$

and its definition is extended to polynomial matrices in a componentwise manner. Observe that if $R(\xi)=R_{0}+R_{1} \xi+\cdots+R_{N} \xi^{N}$, then $\sigma_{+}(R(\xi))=R_{1}+\cdots+R_{L} \xi^{N-1}$, while the second iterate of $\sigma_{+}$, denoted $\sigma_{+}^{2}$, takes the value $\sigma_{+}^{2}(R(\xi))=R_{2}+\cdots+$ $R_{N} \xi^{N-2}$, and similar expressions hold for the $k$ th iterate. It follows from the results of section 6 of [17] that the polynomial matrix

$$
\left[\begin{array}{c}
\sigma_{+}(R(\xi)) \\
\sigma_{+}^{2}(R(\xi)) \\
\vdots \\
\sigma_{+}^{N-1}(R(\xi))
\end{array}\right]
$$

induces a (in general, nonminimal) state map for $R\left(\frac{d}{d t}\right) w=0$. Note that the coefficient matrix of (2.21) is equal to (2.20) up to minus signs, and thus corresponds to an $\tilde{X}$ in a (in general, nonminimal) factorization $\tilde{\Pi}=: \tilde{Y}^{T} \tilde{X}$, with $\tilde{Y}$ an identity matrix up to minus signs. Hence the derivation of $\Pi(\zeta, \eta)$ and its coefficient matrix $\tilde{\Pi}$ can be regarded as a direct and intrinsic "integration by parts" and "BDF" interpretation of the state map construction procedure of [17]. See also [9] for a discussion of the concepts of state and state map from a more algebraic point of view.

Specialization of Theorem 2.5 to input-output systems (1.1) yields the following.

Corollary 2.8. Consider the input-output system (1.1). Define polynomial matrices $X_{y}(\xi), X_{u}(\xi)$ such that

$$
P(-\zeta)-P(\eta)=(\zeta+\eta) Y^{T}(\zeta) X_{y}(\eta), \quad-Q(-\zeta)+Q(\eta)=(\zeta+\eta) Y^{T}(\zeta) X_{u}(\eta)
$$

for some polynomial matrix $Y(\xi)$ (such matrices $X_{y}(\xi), X_{u}(\xi)$, and $Y(\xi)$ always exist); then

$$
x=X_{y}\left(\frac{d}{d t}\right) y+X_{u}\left(\frac{d}{d t}\right) u
$$

defines a state map. 
2.4. Minimality. In this subsection we address the question of which conditions ensure that the state maps obtained by integration by parts are minimal.

Proposition 2.9. Consider $R(-\zeta)-R(\eta)=(\zeta+\eta) \Pi(\zeta, \eta)$, and let $\Pi(\zeta, \eta)=$ $Y^{T}(\zeta) X(\eta)$ be a factorization. Then the state map $x=X\left(\frac{d}{d t}\right) w, x(t) \in \mathbb{R}^{n}$, is minimal if and only if there does not exist a nonzero $f \in \mathbb{R}^{1 \times n}$ such that

$$
f X(\xi)=h(\xi) R(\xi)
$$

for some $h \in \mathbb{R}^{1 \times p}[\xi]$.

Proof. (Only if) Suppose $x=X\left(\frac{d}{d t}\right) w$ is minimal and there exists a nonzero $f \in \mathbb{R}^{1 \times n}$ such that (2.24) holds. Then the image of the state map $x=X\left(\frac{d}{d t}\right) w$ given by

$$
\mathcal{V}:=\left\{v \in \mathbb{R}^{n} \mid \exists w \in \mathcal{B} \text { such that } X\left(\frac{d}{d t}\right) w(0)=v\right\}
$$

is annihilated by $f$; that is, $f v=0$ for all $v \in \mathcal{V}$. Thus the state map is not surjective, contradicting minimality.

(If) The fact that there does not exist a nonzero $f \in \mathbb{R}^{1 \times n}$ such that (2.24) holds implies that the factorization $Y^{T}(\zeta) X(\eta)$ is minimal. Indeed, suppose $Y^{T}(\zeta) X(\eta)$ is not minimal; then there exists a nonzero $f \in \mathbb{R}^{1 \times n}$ such $f X(\xi)=0$. Hence by Lemma 2.6 ,

$$
\left[X\left(\frac{d}{d t}\right) w_{1}(0)=X\left(\frac{d}{d t}\right) w_{2}(0)\right] \Longleftrightarrow\left[w_{1} \sim_{0} w_{2}\right]
$$

Thus the only thing left to be proved for minimality of the state map $x=X\left(\frac{d}{d t}\right) w$ is that it is surjective; that is, $\mathcal{V}$ defined in $(2.25)$ is equal to $\mathbb{R}^{n}$. However, if $\operatorname{dim} \mathcal{V}<n$, then there exists a nonzero constant vector $f \in \mathbb{R}^{1 \times n}$ such that the differential operator $(f X)\left(\frac{d}{d t}\right)$ is zero restricted to $\mathcal{B}$, or, equivalently, such that $f X(\xi)=h(\xi) R(\xi)$ for some $h \in \mathbb{R}^{1 \times p}[\xi]$.

Example 2.10. Consider an input-output system (1.1) without differentiations on $u$,

$$
P\left(\frac{d}{d t}\right) y=u
$$

Computation of a state map is performed by the factorization

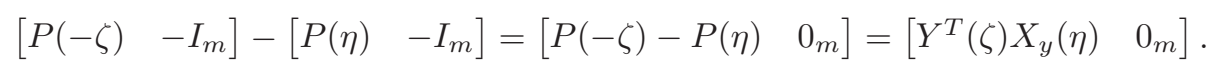

In this case, when the factorization is minimal then also the state map $x=X_{y}\left(\frac{d}{d t}\right) y$ is minimal. Indeed, suppose the state map $x=X_{y}\left(\frac{d}{d t}\right) y$ is not minimal. Then by Proposition 2.9 there would exist a nonzero constant vector $f \in \mathbb{R}^{1 \times n}$ such that

$$
f\left[X_{y}(\xi) \quad 0\right]=h(\xi)\left[P(\xi)-I_{m}\right]
$$

for some $h \in \mathbb{R}^{1 \times m}[\xi]$. However, by the form of this equation we necessarily have $h=0$, which in turn implies by minimality of the factorization that $f=0$.

It follows from the proof of Proposition 2.9 that minimality of the factorization is a necessary condition for minimality of the state map. However, in general it is not sufficient. A simple class of counterexamples is provided by taking $R(\xi)$ to be 
a unimodular polynomial matrix, that is, $R(\xi)$ square and $\operatorname{det} R(\xi)=c$, for some nonzero constant $c$. Then it is clear that the minimal dimension (McMillan degree) of the corresponding system is zero. (In fact, the behavior $\mathcal{B}$ is $\{0\}$.) However, if $R(\xi)$ is not itself a constant matrix, then $\tilde{\Pi}$ will be different from zero, and thus have rank $>0$.

On the other hand, a sufficient (but not necessary) condition for minimality of the state map can be derived as follows. We first recall (see, e.g., [11]) the definition of a row-reduced polynomial matrix. For the $i$ th row of a polynomial matrix $R(\xi)$, we define the row-degree $d_{i}$ as the highest power of $\xi$ appearing in that row. The leading row-coefficient matrix $R_{h r}$ of $R(\xi)$ is defined as the constant matrix, whose $i$ th row consists of the coefficients of $\xi^{d_{i}}$ in the corresponding entry of $R(\xi)$. The polynomial matrix $R(\xi)$ is called row-reduced if $R_{h r}$ has full row-rank.

Proposition 2.11. Consider the system $R\left(\frac{d}{d t}\right) w=0$, with $R$ row-reduced. Let $\Pi(\zeta, \eta)=Y^{T}(\zeta) X(\eta)$ be a minimal factorization; then $x=X\left(\frac{d}{d t}\right) w$ is a minimal state map.

Proof. Recall from the proof of Proposition 2.9 that, in the case of a minimal factorization, minimality of the state map $x=X\left(\frac{d}{d t}\right) w$ with $x \in \mathbb{R}^{n}$ is equivalent to the subspace $\mathcal{V}$ defined in (2.25) having dimension $n$ (equal to the rank of the coefficient matrix $\tilde{\Pi}$ ). First, consider the case when the coefficient matrix of $X$ in the factorization consists of the linearly independent rows of the matrix (2.20); the general case will follow in a straightforward manner.

Assume by contradiction that $\operatorname{dim} \mathcal{V}<n$. Then there exists a nonzero constant vector $f \in \mathbb{R}^{1 \times n}$ such that $f X(\xi)=h(\xi) R(\xi)$ for some $h \in \mathbb{R}^{1 \times p}[\xi]$. We are going to prove that necessarily $h(\xi)$ equals zero. Then $f X(\xi)=0$, and by minimality of the factorization $\Pi(\zeta, \eta)=Y^{T}(\zeta) X(\eta)$ it follows that $f=0$, which yields the desired contradiction.

Denote the row-degrees of $R(\xi)$ by $d_{i}, i=1, \ldots, p$, and without loss of generality, assume that $d_{1}=d_{2}=\cdots=d_{g_{1}}>d_{g_{1}+1} \geq \cdots \geq d_{g_{p}}$. Note that $R$ being row-reduced is equivalent to the predictable degree property (see [11, p. 387]): for every row-vector $h(\xi)$ we have

$$
\operatorname{deg}(h(\xi) R(\xi))=\max _{\substack{i=1, \ldots, p \\ h_{i} \neq 0}}\left\{\operatorname{deg}\left(h_{i}(\xi)\right)+d_{i}\right\}
$$

Note also that every row of $X(\xi)$ has degree less than $d_{1}$, the maximum degree of any row in $R$. Then conclude from $f X(\xi)=h(\xi) R(\xi)$ and the predictable degree property that $h_{i}(\xi)=0, i=1, \ldots, g_{1}$.

Now let $h_{i}(\xi)=0, g_{1}+1 \leq i \leq \bar{g}$, and $h_{\bar{g}+1}(\xi) \neq 0$. The predictable degree property implies that the highest power of $\xi$ in $h(\xi) R(\xi)$ is higher than or equal to $d_{\bar{g}+1}$. It follows then from $f X(\xi)=h(\xi) R(\xi)$ that at least one of the components of $f$ corresponding to the rows of $X$ of degree larger than or equal to $d_{\bar{g}+1}$ must be nonzero. It follows from (2.20) that the rows of $X$ of degree larger than or equal to $d_{\bar{g}+1}$ necessarily correspond to some of the first $\bar{g}$ rows of $R$. Consequently, the highest coefficient vector of $f X(\xi)$ is a linear combination of the highest coefficient vectors of some of the first $\bar{g}$ rows of $R$.

On the other hand, from $f X(\xi)=h(\xi) R(\xi)$ and $h_{i}(\xi)=0$ for $i=1, \ldots, \bar{g}$ it follows that the highest coefficient of $h(\xi) R(\xi)$, and consequently of $f X(\xi)$, is a linear combination of the last $p-\bar{g}$ rows of $R$. However, this implies that a linear combination of the first $\bar{g}$ rows from the highest-coefficient matrix of $R$ equals a linear combination of its last $p-\bar{g}$ rows. By row-reducedness, we conclude that this is 
possible if and only if all of the coefficients of the linear combination equal zero. From this it follows that $h_{i}(\xi)=0$ for all $1 \leq i \leq p$, leading by minimality of the factorization to $f=0$.

In order to prove the case of a general $X$ arising from a minimal factorization, use the fact that any other matrix whose row span equals that of $\tilde{\Phi}$ must differ from the matrix $X$ considered in the previous argument by premultiplication by a nonsingular matrix. This concludes the proof.

Remark 2.12. Recall (see, e.g., $[11,14]$ ) that any polynomial matrix $R(\xi)$ can be transformed into a row-reduced matrix while not changing the behavior defined by it. First of all, for any $R(\xi)$ we can find a unimodular matrix $U(\xi)$ such that $U(\xi) R(\xi)=$ $\left[\begin{array}{c}R^{\prime}(\xi) \\ 0\end{array}\right]$ with $R^{\prime}(\xi)$ full row-rank. Then the behavior defined by $R\left(\frac{d}{d t}\right) w=0$ is the same as that defined by $R^{\prime}\left(\frac{d}{d t}\right) w=0$. Furthermore, for any full-row rank polynomial matrix $R^{\prime}(\xi)$ there exists a unimodular matrix $U^{\prime}(\xi)$ such that $U^{\prime}(\xi) R^{\prime}(\xi)$ is rowreduced. Again, since premultiplication with a unimodular matrix does not change the behavior, the result follows.

Corollary 2.13. If $R$ is row-reduced, then the McMillan degree of $R\left(\frac{d}{d t}\right) w=0$ equals the rank $n$ of the coefficient matrix $\tilde{\Pi}$ of $\Pi(\zeta, \eta)$ in (2.15).

Remark 2.14. The result of Corollary 2.13 provides an alternative way of computing the McMillan degree of a system given in kernel representation $R\left(\frac{d}{d t}\right) w=0$, as compared to the standard way of computing the maximal degree of the nonzero minors of the polynomial matrix $R$. It also implies that in general (without the assumption of row-reducedness) the rank of $\tilde{\Pi}$ is greater than or equal to the McMillan degree. $^{2}$

We illustrate the above theory with a simple example.

Example 2.15. Consider the (uncontrollable) system $\frac{d^{2}}{d t^{2}} w_{1}=\frac{d}{d t} w_{2}$, with corresponding $R(\xi)=\left[\begin{array}{ll}\xi^{2} & -\xi\end{array}\right]$. Compute

$$
R(-\zeta)-R(\eta)=\left[\begin{array}{ll}
\zeta^{2} & \zeta
\end{array}\right]-\left[\begin{array}{ll}
\eta^{2} & -\eta
\end{array}\right]=(\zeta+\eta)\left[\begin{array}{ll}
\zeta-\eta & 1
\end{array}\right] .
$$

A (minimal) factorization of $\Pi(\zeta, \eta):=\left[\begin{array}{ll}\zeta-\eta & 1\end{array}\right]$ can be obtained by (trivially in this case) factorizing its coefficient matrix

$$
\tilde{\Pi}=\left[\begin{array}{cccc}
0 & 1 & -1 & 0 \\
1 & 0 & 0 & 0
\end{array}\right]=\left[\begin{array}{ll}
1 & 0 \\
0 & 1
\end{array}\right]\left[\begin{array}{cccc}
0 & 1 & -1 & 0 \\
1 & 0 & 0 & 0
\end{array}\right]
$$

leading to the factorization

$$
\Pi(\zeta, \eta)=\left[\begin{array}{ll}
\zeta-\eta & 1
\end{array}\right]=\left[\begin{array}{cc}
1 & \zeta
\end{array}\right]\left[\begin{array}{cc}
-\eta & 1 \\
1 & 0
\end{array}\right]=: Y^{T}(\zeta) X(\eta) .
$$

It follows that a state map is given by

$$
\left[\begin{array}{l}
x_{1} \\
x_{2}
\end{array}\right]=\left[\begin{array}{cc}
-\frac{d}{d t} & 1 \\
1 & 0
\end{array}\right]\left[\begin{array}{l}
w_{1} \\
w_{2}
\end{array}\right]=\left[\begin{array}{c}
-\frac{d}{d t} w_{1}+w_{2} \\
w_{1}
\end{array}\right]
$$

which is minimal since $R(\xi)$ is row-reduced. (Indeed, the rank of the coefficient matrix $\tilde{\Pi}$, which is 2, equals the McMillan degree of the system.)

\footnotetext{
${ }^{2}$ An alternative, seemingly unrelated, way of computing the McMillan degree for controllable systems is based on the computation of the rank of the (generalized) Bézoutian (cf. [1]), which can be defined (see [27]) using the two-variable polynomial matrix $R(\zeta) M(-\eta)$, where $w=M\left(\frac{d}{d t}\right) \ell$ is an image representation of the system. Since $R(\zeta) M(\zeta)=0$, it follows that we can write $R(\zeta) M(-\eta)=$ $(\zeta+\eta) \Psi(\zeta, \eta)$, where the coefficient matrix of $\Psi$ is the Bézoutian matrix.
} 
2.5. First-order models. In this subsection we will directly show that the state $x=X\left(\frac{d}{d t}\right) w$ as obtained from a factorization $\Pi(\zeta, \eta)=Y^{T}(\zeta) X(\eta)$ satisfies a set of linear first-order differential-algebraic equations (in accordance with the intuitive notion of state). This follows from the basic formula (2.17). Indeed, for any ordinary solution $w \in \mathcal{B}$, and thus $R\left(\frac{d}{d t}\right) w=0$, this formula yields (by applying the product rule of differentiation to the right-hand side)

$$
\left(Y\left(\frac{d}{d t}\right) \varphi\right)^{T} \frac{d}{d t} x=-\left(\frac{d}{d t}\left[Y\left(\frac{d}{d t}\right) \varphi\right]\right)^{T} x+w^{T}\left(R^{T}\left(-\frac{d}{d t}\right) \varphi\right)
$$

for all $\varphi$. Denote $Y(\xi)=Y_{0}+Y_{0} \xi+\cdots+Y_{N-1} \xi^{N-1}$. Then this expression can be written explicitly as

$$
\begin{gathered}
{\left[\begin{array}{llll}
\varphi^{T} & \varphi^{(1) T} & \cdots & \varphi^{(N-1) T}
\end{array}\right]\left[\begin{array}{c}
Y_{0}^{T} \\
\vdots \\
Y_{N-1}^{T}
\end{array}\right] \frac{d}{d t} x} \\
=-\left[\begin{array}{llll}
\varphi^{(1) T} & \varphi^{(2) T} & \cdots & \varphi^{(N) T}
\end{array}\right]\left[\begin{array}{c}
Y_{0}^{T} \\
\vdots \\
Y_{N-1}^{T}
\end{array}\right] x \\
+\left[\begin{array}{llll}
\varphi^{T} & \varphi^{(1) T} & \cdots & \varphi^{(N) T}
\end{array}\right]\left[\begin{array}{c}
R_{0} \\
-R_{1} \\
\vdots \\
\\
(-1)^{N} R_{N}
\end{array}\right] w
\end{gathered}
$$

for all row vectors $\left[\begin{array}{llll}\varphi^{T} & \varphi^{(1) T} & \cdots & \varphi^{(N) T}\end{array}\right]$, or more compactly,

$$
\left[\begin{array}{lll}
\varphi^{T} & \cdots & \varphi^{(N) T}
\end{array}\right]\left(\left[\begin{array}{c}
Y_{0}^{T} \\
\vdots \\
Y_{N-1}^{T} \\
0
\end{array}\right] \frac{d}{d t} x+\left[\begin{array}{c}
0 \\
Y_{0}^{T} \\
\vdots \\
Y_{N-1}^{T}
\end{array}\right] x+\left[\begin{array}{c}
-R_{0} \\
R_{1} \\
\vdots \\
-(-1)^{N} R_{N}
\end{array}\right] w\right)=0 .
$$

From the arbitrariness of the test function $\varphi$, and hence the arbitrariness of the row vector $\left[\begin{array}{llll}\varphi^{T} & \varphi^{(1) T} & \cdots & \varphi^{(N) T}\end{array}\right]$, it thus follows that

$$
\left[\begin{array}{c}
Y_{0}^{T} \\
\vdots \\
Y_{N-1}^{T} \\
0
\end{array}\right] \frac{d}{d t} x+\left[\begin{array}{c}
0 \\
Y_{0}^{T} \\
\vdots \\
Y_{N-1}^{T}
\end{array}\right] x+\left[\begin{array}{c}
-R_{0} \\
R_{1} \\
\vdots \\
-(-1)^{N} R_{N}
\end{array}\right] w=0
$$

Note that (2.30) constitutes a state representation of the differential-algebraic form $E \frac{d}{d t} x+F x+G w=0$. If the factorization $\Pi(\zeta, \eta)=Y^{T}(\zeta) X(\eta)$ is chosen to be minimal, then

$$
\left[\begin{array}{c}
Y_{0}^{T} \\
\vdots \\
Y_{N-1}^{T}
\end{array}\right]
$$


has full column rank, and thus has a left inverse $L$. Premultiplying (2.30) by $\left[\begin{array}{ll}L & 0 \\ 0 & I_{p}\end{array}\right]$ then yields the equivalent representation

$$
\begin{aligned}
\frac{d}{d t} x & =-L\left[\begin{array}{c}
0 \\
Y_{0}^{T} \\
\vdots \\
Y_{N-2}^{T}
\end{array}\right] x+L\left[\begin{array}{c}
R_{0} \\
-R_{1} \\
\vdots \\
(-1)^{N-1} R_{N-1}
\end{array}\right] w, \\
0 & =-Y_{N-1}^{T} x+(-1)^{N} R_{N} w .
\end{aligned}
$$

This is a representation of the form $\frac{d}{d t} x=F x+G w, 0=H x+K w$, often called an output nulling representation. Evidently, different factorizations of $\tilde{\Pi}$ yield different state maps and consequently different state equations (2.30), (2.31); in a future paper we will further explore the resulting state space realizations.

2.6. State maps for image representations. A linear system in image representation ${ }^{3}$ is given by higher-order differential equations of the form

$$
w=M\left(\frac{d}{d t}\right) \ell
$$

where $\ell \in \mathcal{L}_{1}^{\text {loc }}\left(\mathbb{R}, \mathbb{R}^{k}\right)$ denotes a vector function of auxiliary, or latent, variables, and $M(\xi)$ is an $q \times k$ polynomial matrix. Note that (2.32) defines two behaviors: the full behavior,

$$
\mathcal{B}_{\text {full }}:=\left\{(w, \ell) \in \mathcal{L}_{1}^{\text {loc }}\left(\mathbb{R}, \mathbb{R}^{(q+k)} \mid(2.32) \text { holds weakly }\right\},\right.
$$

and the external behavior,

$$
\mathcal{B}_{\text {ext }}:=\left\{w \in \mathcal{L}_{1}^{\text {loc }}\left(\mathbb{R}, \mathbb{R}^{q}\right) \mid \exists \ell \in \mathcal{L}_{1}^{\text {loc }}\left(\mathbb{R}, \mathbb{R}^{k}\right) \text { such that (2.32) holds weakly }\right\} .
$$

Systems in image representation will naturally come up in sections 3 and 4 . It is well known [14] that any system $R\left(\frac{d}{d t}\right) w=0$ with behavior $\mathcal{B}$ can be written in image representation $(2.32)$ (in the sense that $\mathcal{B}_{\text {ext }}=\mathcal{B}$ ) if and only if it is controllable (in the behavioral sense), or equivalently, the polynomial matrix $R(s), s \in \mathbb{C}$, has constant rank. Thus systems in image representation are necessarily controllable.

The construction of a state map for the full behavior $\mathcal{B}_{\text {full }}$ directly follows from the construction of state maps for systems given in kernel representation (2.1) as above. Indeed, the full behavior is given as the set of weak solutions (in $w$ and $\ell$ )

$$
\left[I_{q}-M\left(\frac{d}{d t}\right)\right]\left[\begin{array}{l}
w \\
\ell
\end{array}\right]=0 .
$$

Thus a state map can be constructed by factorizing the two-variable polynomial matrix $\Pi(\zeta, \eta)$ defined as

$$
\begin{aligned}
(\zeta+\eta) \Pi(\zeta, \eta) & :=\left[\begin{array}{ll}
I_{q} & -M(-\zeta)
\end{array}\right]-\left[\begin{array}{ll}
I_{q} & -M(\eta)
\end{array}\right] \\
& =\left[\begin{array}{ll}
0_{q} & -M(-\zeta)+M(\eta)
\end{array}\right] .
\end{aligned}
$$

\footnotetext{
${ }^{3}$ Correspondingly, (2.1) is often referred to as a kernel representation.
} 
Obviously, any factorization of $\Pi(\zeta, \eta)$ is of the form

$$
\Pi(\zeta, \eta)=Y_{M}^{T}(\zeta)\left[\begin{array}{ll}
0_{q} & \left.X_{M}(\eta)\right]
\end{array}\right.
$$

where $Y_{M}^{T}(\zeta) X_{M}(\eta)$ is a factorization of $\Pi_{M}(\zeta, \eta)$, defined by $(\zeta+\eta) \Pi_{M}(\zeta, \eta):=$ $-M(-\zeta)+M(\eta)$. In particular, the state map for the full behavior is a function of only the latent variables $\ell$.

Somewhat contrary to the case of kernel representations, minimality of this state map simply corresponds to minimality of the factorization $\Pi_{M}(\zeta, \eta)=Y_{M}^{T}(\zeta) X_{M}(\eta)$, as is formulated in the next proposition.

Proposition 2.16. Consider the full behavior of the image representation (2.32). Any factorization

$$
\Pi_{M}(\zeta, \eta)=\frac{-M(-\zeta)+M(\eta)}{\zeta+\eta}=Y_{M}^{T}(\zeta) X_{M}(\eta)
$$

defines a state map $x=X_{M}\left(\frac{d}{d t}\right) \ell$ for the full behavior, which is minimal if and only if the factorization is minimal.

Proof. The only thing left to be proved is the "if" part concerning minimality. This follows, however, from similar reasoning as in Example 2.10. Indeed, consider a minimal factorization $\Pi_{M}(\zeta, \eta)=Y_{M}^{T}(\zeta) X_{M}(\eta)$, yielding a state map $X(\xi)=\left[\begin{array}{ll}0 & X_{M}(\xi)\end{array}\right]$, where 0 is an $n \times q$ zero-matrix. Suppose this state map is not minimal. Then, by Proposition 2.9, there would exist a nonzero constant vector $f \in \mathbb{R}^{1 \times n}$ such that

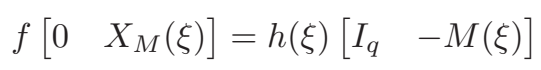

for some $h \in \mathbb{R}^{1 \times q}[\xi]$. However, by the form of this equation it follows that we necessarily have $h=0$, which in turn implies by minimality of the factorization the contradiction $f=0$.

State maps for the external behavior $\mathcal{B}_{\text {ext }}$ can be directly obtained from state maps for the full behavior $\mathcal{B}_{\text {full }}$ once the latent variables $\ell$ can be expressed in the external variables $w$. It is well known (see [14]) that for every image representation (2.32) we can replace the polynomial matrix $M(\xi)$ by a polynomial matrix $M^{\prime}(\xi)$ such that $M^{\prime}(s)$ has full column-rank for all $s \in \mathbb{C}$, while not changing the external behavior of the image representation. ${ }^{4}$ For such a, possibly adapted, image representation we have the following.

Proposition 2.17. Consider (2.32), where $M(s)$ has full column-rank for all $s \in \mathbb{C}$, and consider the full (2.33) and external (2.34) behaviors associated with (2.32). Then

$$
\left[\left(w_{1}, \ell_{1}\right) \sim_{0}\left(w_{2}, \ell_{2}\right) \text { in }(2.33)\right] \Longleftrightarrow\left[w_{1} \sim_{0} w_{2} \text { in }(2.34)\right] .
$$

Let $L(\xi)$ be such that

$$
L(\xi) M(\xi)=I,
$$

and let $x=X_{M}\left(\frac{d}{d t}\right)$ l be a (minimal) state map for the full behavior $\mathcal{B}_{\text {full }}$. Then $x=X_{M}\left(\frac{d}{d t}\right) L\left(\frac{d}{d t}\right) w$ is a (minimal) state map for the external behavior $\mathcal{B}_{\text {ext }}$. In particular, the McMillan degrees of the full and the external behaviors are equal.

\footnotetext{
${ }^{4}$ Such an image representation is called observable, since the latent variables $\ell$ are uniquely determined by the external variables $w$. Indeed, since $M^{\prime}(s)$ has constant rank for all $s \in \mathbb{C}$, there exists a polynomial matrix $L(\xi)$ such that $L(\xi) M^{\prime}(\xi)=I$, and hence $\ell=L\left(\frac{d}{d t}\right) M^{\prime}\left(\frac{d}{d t}\right) \ell=L\left(\frac{d}{d t}\right) w$.
} 
Proof. The implication $\Rightarrow$ is obvious. In order to prove the converse implication, observe that the latent variable trajectory $\ell$ is uniquely determined by $w$ :

$$
\left[\left(w_{1}, \ell_{1}\right),\left(w_{2}, \ell_{2}\right) \in \mathcal{B}_{\text {full }}\right] \text { and }\left[w_{1}=w_{2}\right] \Longrightarrow\left[\ell_{1}=\ell_{2}\right]
$$

Now assume that $w_{1}, w_{2} \in \mathcal{B}_{\text {ext }}$ are infinitely differentiable trajectories which are equivalent at zero in the external behavior, i.e., $w_{1} \wedge_{0} w_{2} \in \mathcal{B}_{\text {ext }}$. Let $\ell$ be the latent variable corresponding to $w_{1} \wedge_{0} w_{2}$, i.e., $\left(w_{1} \wedge_{0} w_{2}, \ell\right) \in \mathcal{B}_{\text {full }}$. Proceed now as in the proof of Lemma 2.6 and integrate by parts along $\left(w_{1} \wedge_{0} w_{2}, \ell\right)$ on $(-\infty, 0)$ and $(0,+\infty)$, obtaining algebraic conditions on $\ell$ and its derivatives on the left and the right of zero. Now apply observability to conclude that the restriction of $\ell$ to $(-\infty, 0)$ coincides with $\ell_{1}$, the latent variable trajectory such that $w_{1}=R\left(\frac{d}{d t}\right) \ell_{1}$, and that the restriction of $\ell$ to $(0,+\infty)$ coincides with $\ell_{2}$, the latent variable trajectory such that $w_{2}=R\left(\frac{d}{d t}\right) \ell_{2}$. Consequently, the algebraic conditions on $\ell$ and its derivatives on the left and the right of zero can be written in terms of $\ell_{1}$, respectively, $\ell_{2}$. These conditions are readily seen to be the same as the concatenability conditions for the full trajectories $\left(w_{1}, \ell_{1}\right)$ and $\left(w_{2}, \ell_{2}\right)$ obtained by integration by parts on the full trajectories. The rest of the claims follow immediately.

Remark 2.18. One implication in the result of Proposition 2.17 is straightforward: if two trajectories are concatenable in the full behavior, then their projections on the external variables are concatenable in the external behavior. The converse implication is interesting since it shows that if two trajectories are concatenable at time $t=0$ in the external behavior, then the full trajectories corresponding to them are also concatenable at zero, producing an admissible full trajectory. (Note that this is in general not true for general representations involving latent variables, as discussed in section 7 of [17].)

3. Adjoint systems. Consider again the basic integration by parts formula (2.9). For any $\mathcal{C}^{\infty}$ function $w \in \mathcal{B}$ it holds that $R\left(\frac{d}{d t}\right) w=0$, and thus we obtain from (2.9) the equality

$$
w^{T}(t) R^{T}\left(-\frac{d}{d t}\right) \varphi(t)=\frac{d}{d t} B_{\Pi}(\varphi, w)(t) .
$$

This formula is used to define (see also [6]) the adjoint system as the system given in image representation as

$$
w_{a}=R^{T}\left(-\frac{d}{d t}\right) \varphi
$$

where $w_{a}(t)$ belongs to $\mathcal{W}^{*}=\mathbb{R}^{q}$, the dual space of $\mathcal{W}$. This leads to the following relation between the trajectories of the original system $R\left(\frac{d}{d t}\right) w=0$ and those of its adjoint system (3.2):

$$
w^{T} w_{a}=\frac{d}{d t} B_{\Pi}(\varphi, w)
$$

Since the adjoint system is given in image representation, it is automatically controllable.

Remark 3.1. It can be seen that (3.3) uniquely defines the adjoint system in the following sense. Suppose there exists a trajectory $w_{a}^{\prime}$ such that for some other bilinear differential form $B_{\Pi^{\prime}}(\varphi, w)$,

$$
w^{T} w_{a}^{\prime}=\frac{d}{d t} B_{\Pi^{\prime}}(\varphi, w)
$$

Copyright (C) by SIAM. Unauthorized reproduction of this article is prohibited. 
for all $w \in \mathcal{B}$. Then, following an argument in [10], by subtracting (3.4) from (3.3) it follows that

$$
w^{T}\left(w-w_{a}^{\prime}\right)=\frac{d}{d t}\left[B_{\Pi}(\varphi, w)-B_{\Pi^{\prime}}(\varphi, w)\right]
$$

for all $w \in \mathcal{B}$. Since the left-hand side does not depend on the derivative of $N$ th order $w^{(N)}$ this implies that $B_{\Pi}(\varphi, w)-B_{\Pi}^{\prime}(\varphi, w)$ does not depend on $w^{(N-1)}$. However, since the left-hand side also does not depend on $w^{(N-1)}$, this implies that $B_{\Pi}(\varphi, w)-B_{\Pi^{\prime}}(\varphi, w)$ also does not depend on $w^{(N-2)}$. This argument may be repeated to show that the bilinear differential form $B_{\Pi}(\varphi, w)-B_{\Pi^{\prime}}(\varphi, w)$ does not depend on $w^{(N-1)}, w^{(N-2)}, \ldots, w$, and hence (by linearity and arbitrariness of $\varphi$ ) is equal to zero. It follows that $w_{a}=w_{a}^{\prime}$, and thus the set of trajectories of the adjoint system is uniquely defined by (3.3).

Remark 3.2. The above definition differs from the classical definition of the adjoint system as in, e.g., [10]. In fact, for a system of differential equations $R\left(\frac{d}{d t}\right) w=$ 0 , where $R(\xi)$ is assumed to be a square polynomial matrix, the adjoint system is classically defined as the set of differential equations $R^{T}\left(-\frac{d}{d t}\right) \varphi=0$ in the vector $\varphi$.

As shown in Theorem 2.5, the right-factor of a factorization $\Pi(\zeta, \eta)=Y^{T}(\zeta) X(\eta)$ defines a state map for the system $R\left(\frac{d}{d t}\right) w=0$. It turns out that the left-factor $Y(\xi)$ is associated with a state map for the full behavior of the adjoint system.

TheOrem 3.3. Let $\Pi(\zeta, \eta)$ be defined as in (2.11), and let $\Pi(\zeta, \eta)=Y^{T}(\zeta) X(\eta)$ be a factorization. Then the map $x_{a}:=Y\left(\frac{d}{d t}\right) \varphi$ acting on the latent variable $\varphi$ is a state map for the full behavior.

Proof. The claim follows in a straightforward manner from the argument used in the proof of Proposition 2.17.

Remark 3.4. In section 2 we defined state maps as differential operators that act on the external variables of a system, thus producing a state vector. When considering representations involving latent variables, it is also possible (see section 7 of [17]) to define state maps also as acting on the full trajectories to produce state variables for the external behavior. In this broader sense, the state map $x_{a}:=Y\left(\frac{d}{d t}\right) \varphi$ defined in Theorem 3.3 acting on the latent variable $\varphi$ is a state map also for the external behavior of (3.2), for the same reason mentioned at the beginning of Remark 2.18.

From (3.3) we conclude that the state $x_{a}=Y\left(\frac{d}{d t}\right) \varphi$ of the adjoint system satisfies (see also $[27$, eq. (10.3)] or $[6$, eq. (2.6)])

$$
w_{a}^{T} w=\frac{d}{d t} x_{a}^{T} x .
$$

It is important to note that the adjoint system is fully determined by the controllable part of the system $R\left(\frac{d}{d t}\right) w=0$, which is defined as follows. First, without loss of generality we may assume that $R(\xi)$ has full row-rank; cf. Remark 2.12. Next, any full row-rank matrix $R(\xi)$ can be factorized as

$$
R(\xi)=P(\xi) R_{c}(\xi)
$$

where $R_{c}(s)$ has full row-rank for all $s \in \mathbb{C}$, and $P(\xi)$ is a square polynomial matrix such that $\operatorname{det} P(\xi)$ is different from the zero-polynomial (see, e.g., [14]). Then

$$
\mathcal{B}_{c}:=\left\{w \in \mathcal{L}_{1}^{\text {loc }}\left(\mathbb{R}, \mathbb{R}^{q}\right) \mid w \text { is a weak solution of } R_{c}\left(\frac{d}{d t}\right) w(t)=0\right\}
$$


obviously satisfies $\mathcal{B}_{c} \subset \mathcal{B}$ and constitutes the controllable part of the system. Since $P$ is nonsingular, the differential operator $P^{T}\left(-\frac{d}{d t}\right)$ is surjective (cf. [14]), and consequently the time-functions $\varphi_{c}:=P^{T}\left(-\frac{d}{d t}\right) \varphi$ are arbitrary time-functions, so that the adjoint system is also given as

$$
w_{a}=R_{c}^{T}\left(-\frac{d}{d t}\right)\left[P^{T}\left(-\frac{d}{d t}\right) \varphi\right]=R_{c}^{T}\left(-\frac{d}{d t}\right) \varphi_{c}
$$

which is indeed involving only the controllable part of the system. Since $R_{c}(s)$ is surjective for all $s \in \mathbb{C}$, application of Proposition 2.17 yields the following corollary.

COROLlary 3.5. Consider the adjoint system given in image representation,

$$
w_{a}=R_{c}^{T}\left(-\frac{d}{d t}\right) \varphi_{c}
$$

Let $L_{c}(\xi)$ be such that

$$
L_{c}(\xi) R_{c}^{T}(-\xi)=I
$$

and let $x=Y_{c}\left(\frac{d}{d t}\right) \varphi_{c}$ be a (minimal) state map for the full behavior $\mathcal{B}_{\text {full }}$ of the adjoint system. Then $x=Y_{c}\left(\frac{d}{d t}\right) L_{c}\left(\frac{d}{d t}\right) w$ is a (minimal) state map for the external behavior $\mathcal{B}_{\text {ext }}$ of the adjoint system. In particular, the McMillan degrees of the full and the external behaviors of the adjoint system are equal.

As a consequence we can derive the following behavioral characterization of the adjoint system, extending the one given in $[13,27]$ for controllable systems.

Proposition 3.6. Consider the system $R\left(\frac{d}{d t}\right) w=0$. Then the external behavior $\mathcal{B}_{a}$ of the adjoint system (3.2) equals

$$
\begin{aligned}
& \left\{w_{a} \in \mathcal{L}_{1}^{\text {loc }}\left(\mathbb{R}, \mathbb{R}^{q}\right) \mid \int_{-\infty}^{\infty} w_{a}^{T}(t) w(t) d t=0 \quad \forall w \in \mathcal{B}_{c} \text { with compact support }\right\} \\
& =\left\{w_{a} \in \mathcal{L}_{1}^{\text {loc }}\left(\mathbb{R}, \mathbb{R}^{q}\right) \mid \int_{-\infty}^{\infty} w_{a}^{T}(t) w(t) d t=0 \quad \forall w \in \mathcal{B} \text { with compact support }\right\} .
\end{aligned}
$$

Proof. Without loss of generality $R(\xi)=P(\xi) R_{c}(\xi)$ is given as in (3.7). Since $R_{c}\left(\frac{d}{d t}\right) w=0$ is a controllable system, it has been shown in $[13,27]$ that the behavior $\mathcal{B}_{a}$ of the adjoint system (3.5) equals the expression in the first line of (3.11). On the other hand, all trajectories of compact support in $\mathcal{B}$ are actually (cf. [14]) contained in $\mathcal{B}_{c}$, and hence the result follows.

4. State maps for Hamiltonian and time-reversible systems. Throughout subsections 4.1 and 4.2 we consider controllable systems $R\left(\frac{d}{d t}\right) w=0$, or equivalently, in image representation $w=M\left(\frac{d}{d t}\right) \ell$, where $\operatorname{im} M(s)=\operatorname{ker} R(s), s \in \mathbb{C}$, and, without loss of generality, $M(s)$ has full column-rank for all $s \in \mathbb{C}$.

Suppose that $\mathcal{W}=\mathbb{R}^{q}$ is given as $\mathcal{W}=\mathcal{U} \times \mathcal{U}^{*}$ for some linear space $\mathcal{U}:=\mathbb{R}^{m}$ (hence $q=2 m$ ). Such a space $\mathcal{W}=\mathcal{U} \times \mathcal{U}^{*}$ is endowed with two canonical nondegenerate bilinear forms. The first is the skew-symmetric bilinear form defined by

$$
\left[\left(u_{1}, y_{1}\right),\left(u_{2}, y_{2}\right)\right]:=\left\langle y_{1} \mid u_{2}\right\rangle-\left\langle y_{2} \mid u_{1}\right\rangle
$$

for $u_{1}, u_{2} \in \mathcal{U}$, and $y_{1}, y_{2} \in \mathcal{U}^{*}$, where $\langle\cdot \mid \cdot\rangle$ denotes the duality product between $\mathcal{U}$ and its dual $\mathcal{U}^{*}$. This is nothing else than the standard symplectic form given in 
matrix representation by

$$
J_{e}=\left[\begin{array}{cc}
0_{m} & I_{m} \\
-I_{m} & 0_{m}
\end{array}\right]
$$

The second canonical bilinear form on $\mathcal{W}=\mathcal{U} \times \mathcal{U}^{*}$ is the symmetric bilinear form defined by

$$
\left\langle\left(u_{1}, y_{1}\right),\left(u_{2}, y_{2}\right)\right\rangle:=\left\langle y_{1} \mid u_{2}\right\rangle+\left\langle y_{2} \mid u_{1}\right\rangle
$$

which has the matrix representation

$$
Q_{e}=\left[\begin{array}{ll}
0_{m} & I_{m} \\
I_{m} & 0_{m}
\end{array}\right]
$$

Both canonical forms give rise to a definition of a Hamiltonian system: the first is that of a self-adjoint Hamiltonian system (closely related to the input-output Hamiltonian systems introduced in [2, 5] and further studied, e.g., in [18, 19]), and the second is that of a (conservative) port-Hamiltonian system (as introduced in [7, 12, 23]). In both cases we will show how the state map construction developed in section 2 translates into the explicit construction of a symplectic, respectively, energy quadratic, form on the state space.

4.1. Self-adjoint Hamiltonian systems. The skew-symmetric bilinear form $[\cdot, \cdot]$ with matrix representation $J_{e}$ on $\mathcal{W}=\mathbb{R}^{2 m}$ gives rise to the following skewsymmetric bilinear form on the set $\mathcal{C}\left(\mathbb{R}, \mathbb{R}^{2 m}\right)$ of piecewise right-continuous functions ${ }^{5}$ $w: \mathbb{R} \rightarrow \mathbb{R}^{2 m}$ with compact support:

$$
\left[\left[w_{1}, w_{2}\right]\right]:=\int_{-\infty}^{\infty} w_{1}^{T}(t) J_{e} w_{2}(t) d t .
$$

This is again a nondegenerate form, in the sense that if $\left[\left[w_{1}, w_{2}\right]\right]=0$ for all compact support $w_{1}$ (resp., $w_{2}$ ), then $w_{2}=0$ (resp., $w_{1}=0$ ). Thus it defines a symplectic form on $\mathcal{C}\left(\mathbb{R}, \mathbb{R}^{2 m}\right)$. Recall that a subspace $\mathcal{L}$ of a linear space $\mathcal{V}$ with symplectic form $\omega$ is called Lagrangian if $\mathcal{L}=\mathcal{L}^{\perp}$, where ${ }^{\perp}$ denotes the orthogonal complement with respect to the symplectic form $\omega$. Equivalently, $\mathcal{L}$ is Lagrangian if $\omega$ is zero when restricted to $\mathcal{L}$, and moreover $\mathcal{L}$ is maximal with respect to this property. This leads to the following behavioral definition of a self-adjoint Hamiltonian system as initially developed in $[6,19,20,21]$.

DEFINITION 4.1. The behavior $\mathcal{B}$ defines a self-adjoint Hamiltonian system if $\mathcal{B}$ is a Lagrangian subspace with respect to the symplectic form $[[\cdot, \cdot]]$ defined in $(4.5)$ on $\mathcal{C}\left(\mathbb{R}, \mathbb{R}^{2 m}\right)$.

Hence the system $w=M\left(\frac{d}{d t}\right) \ell$ is self-adjoint Hamiltonian if

$$
\int_{-\infty}^{\infty} w_{1}^{T}(t) J_{e} w_{2}(t) d t=\int_{-\infty}^{\infty}\left(M\left(\frac{d}{d t}\right) \ell_{1}(t)\right)^{T} J_{e} M\left(\frac{d}{d t}\right) \ell_{2}(t)=0
$$

for all smooth $\ell_{1}, \ell_{2}$ of compact support, and furthermore the system is maximal with regard to this property. It follows from [19, 6] (see also [27, Theorem 3.1] for similar

\footnotetext{
${ }^{5}$ Note that we have replaced the function class $\mathcal{L}_{1}^{\text {loc }}\left(\mathbb{R}, \mathbb{R}^{q}\right)$, as used up to now in the definition of behaviors, by the smaller class of piecewise right-continuous functions - the reason being that we need the expressions (4.5) to be well defined.
}

Copyright $@$ by SIAM. Unauthorized reproduction of this article is prohibited. 
developments) that $w=M\left(\frac{d}{d t}\right) \ell$ is self-adjoint Hamiltonian if and only if

$$
M^{T}(-s) J_{e} M(s)=0, \quad \operatorname{dimim} M(s)=m\left(=\frac{1}{2} \operatorname{dim} \mathcal{W}\right) \quad \forall s \in \mathbb{C} .
$$

The equality (4.7) means that $M^{T}(\zeta) J_{e} M(\eta)$ is zero whenever $\zeta+\eta=0$. Hence (4.7) is equivalent to the existence of a two-variable polynomial matrix $\Phi(\zeta, \eta)$ such that

$$
M^{T}(\zeta) J_{e} M(\eta)=(\zeta+\eta) \Phi(\zeta, \eta) .
$$

Since by (4.8) we have that $\Phi(\zeta, \eta)=-\Phi^{T}(\eta, \zeta)$, the coefficient matrix $\tilde{\Phi}$ of $\Phi(\zeta, \eta)$ is skew-symmetric, and consequently it admits a factorization $\tilde{\Phi}=\tilde{Z}^{T} J_{i} \tilde{Z}$ for some full row-rank matrix $\tilde{Z}$ and some nonsingular skew-symmetric $n \times n$ matrix $J_{i}$, where $n$ is necessarily even-dimensional. This factorization of $\tilde{\Phi}$ induces a minimal factorization

$$
\Phi(\zeta, \eta)=Z^{T}(\zeta) J_{i} Z(\eta), \quad J_{i}=-J_{i}^{T} .
$$

Theorem 4.2. Let $w=M\left(\frac{d}{d t}\right) \ell$ with external behavior $\mathcal{B}$ be a self-adjoint Hamiltonian system. Consider the minimal factorization (4.9). Then $x=Z\left(\frac{d}{d t}\right) \ell$ is a minimal state map as in section 2, with McMillan degree equal to $n$ (even). Furthermore, for every $w_{i}=M\left(\frac{d}{d t}\right) \ell_{i} \in \mathcal{B}$ with associated state trajectories $x_{i}:=$ $Z\left(\frac{d}{d t}\right) \ell_{i}, i=1,2$, it holds that

$$
\frac{d}{d t} x_{1}^{T} J_{i} x_{2}=w_{1}^{T} J_{e} w_{2}
$$

Proof. Start again from the minimal state map construction for the system $w=$ $M\left(\frac{d}{d t}\right) \ell$, based on the two-variable polynomial matrix equality

$$
-M(-\zeta)+M(\eta)=(\zeta+\eta) \Pi_{M}(\zeta, \eta)
$$

and a minimal factorization $\Pi_{M}(\zeta, \eta)=Y_{M}^{T}(\zeta) X_{M}(\eta)$ (see subsection 2.6). Substitution of $M(\eta)=M(-\zeta)+(\zeta+\eta) \Pi_{M}(\zeta, \eta)$ in (4.8) leads to (since $M(\zeta)^{T} J_{e} M(-\zeta)=0$ )

$$
(\zeta+\eta) M^{T}(\zeta) J_{e} \Pi_{M}(\zeta, \eta)=(\zeta+\eta) \Phi(\zeta, \eta),
$$

and hence

$$
M^{T}(\zeta) J_{e} \Pi_{M}(\zeta, \eta)=\Phi(\zeta, \eta) .
$$

Substituting (4.9) in (4.11) and equating the powers of $\zeta$ and $\eta$ in the resulting equation

$$
M^{T}(\zeta) J_{e} Y_{M}^{T}(\zeta) X_{M}(\eta)=Z^{T}(\zeta) J_{i} Z(\eta),
$$

it follows that (up to premultiplication by an invertible matrix; cf. Proposition 2.4) $X_{M}(\eta)=Z(\eta)$. Consequently, $x=Z\left(\frac{d}{d t}\right) \ell$ is a minimal state map for the full behavior of $w=M\left(\frac{d}{d t}\right) \ell$.

In order to prove the last part, combine (4.8) and (4.9) into

$$
M^{T}(\zeta) J_{e} M(\eta)=(\zeta+\eta) \Phi(\zeta, \eta)=(\zeta+\eta) Z^{T}(\zeta) J_{i} Z(\eta) .
$$

The time-domain translation of this equality is that for every $w_{i}=M\left(\frac{d}{d t}\right) \ell_{i}$ and corresponding state trajectories $x_{i}=Z\left(\frac{d}{d t}\right) \ell_{i}, i=1,2$, the equality (4.10) holds.

Copyright (c) by SIAM. Unauthorized reproduction of this article is prohibited. 
Example 4.3. Consider the case $q=2$, with the system given by $p\left(\frac{d}{d t}\right) y=q\left(\frac{d}{d t}\right) u$, where $p(\xi)$ and $q(\xi)$ are coprime polynomials, with image representation

$$
\left[\begin{array}{l}
u \\
y
\end{array}\right]=\left[\begin{array}{l}
p\left(\frac{d}{d t}\right) \\
q\left(\frac{d}{d t}\right)
\end{array}\right] \ell .
$$

This defines a self-adjoint Hamiltonian system if and only if for all $s \in \mathbb{C}$

$$
-p(-s) q(s)+q(-s) p(s)=0 .
$$

A minimal state map is obtained by a minimal factorization ${ }^{6}$ (cf. (4.11) and (4.9))

$$
-p(\zeta) q(\eta)+q(\zeta) p(\eta)=(\zeta+\eta) \Phi(\zeta, \eta)=(\zeta+\eta) Z^{T}(\zeta) J_{i} Z(\eta)
$$

where $J_{i}$ is a skew-symmetric matrix of full-rank. For instance, $p(s)=s^{2}$ and $q(s)=1$ lead to the minimal factorization

$$
-\zeta^{2}+\eta^{2}=(\zeta+\eta)(-\zeta+\eta)=\left[\begin{array}{cc}
1 & \zeta
\end{array}\right]\left[\begin{array}{cc}
0 & 1 \\
-1 & 0
\end{array}\right]\left[\begin{array}{l}
1 \\
\eta
\end{array}\right]
$$

defining the minimal state map

$$
x=\left[\begin{array}{c}
1 \\
\frac{d}{d t}
\end{array}\right] l=\left[\begin{array}{cc}
0 & 1 \\
0 & \frac{d}{d t}
\end{array}\right]\left[\begin{array}{l}
u \\
y
\end{array}\right] .
$$

Remark 4.4. An input-output system given in transfer matrix format $y(s)=$ $G(s) u(s), u \in \mathcal{U}, y \in \mathcal{U}^{*}$, is self-adjoint Hamiltonian if and only if

$$
G(s)=G^{T}(-s) .
$$

This is the starting point taken in $[5,19,18]$, where the internal symplectic form $J_{i}$ is obtained as a consequence of the state space isomorphism theorem, contrary to the explicit construction of $J_{i}$ in (4.9); see also [21, 20] for related results.

Remark 4.5. The terminology "self-adjoint Hamiltonian" can be understood as follows. Consider the controllable behavior $\mathcal{B}$ on $\mathcal{W}$ with symplectic form $J_{e}$, together with its adjoint system behavior $\mathcal{B}_{a}$. Then $J_{e}$ induces an invertible map (denoted by the same symbol) $J_{e}: \mathcal{W} \rightarrow \mathcal{W}^{*}$, and $\mathcal{B}$ is self-adjoint Hamiltonian if and only if (see also $[6,19])$

$$
J_{e} \mathcal{B}=\mathcal{B}_{a}
$$

Furthermore, the symplectic form $J_{i}$ induces an invertible map $J_{i}: \mathcal{X} \rightarrow \mathcal{X}^{*}$. Comparing (4.10) with the basic formula (3.6) for the adjoint system, we have the correspondence $w_{a}=J_{e} w, x_{a}=J_{i} x$. Hence, the full behavior $\mathcal{B}_{\text {full }}$ of a self-adjoint Hamiltonian system satisfies

$$
\left[\begin{array}{cc}
J_{e} & 0 \\
0 & J_{i}
\end{array}\right] \mathcal{B}_{\text {full }}=\left(\mathcal{B}_{a}\right)_{\text {full }}
$$

\footnotetext{
${ }^{6}$ Note that $\Phi(\zeta, \eta)$ is close to (although different from!) the classical Bézoutian of the polynomials $p(\xi), q(\xi)$.
}

Copyright (c) by SIAM. Unauthorized reproduction of this article is prohibited. 
4.2. Conservative port-Hamiltonian systems. The nondegenerate bilinear form $Q_{e}$ defined in (4.4) gives rise to the following symmetric bilinear form on the set $\mathcal{C}\left(\mathbb{R}, \mathbb{R}^{2 m}\right)$ of piecewise right-continuous functions $w: \mathbb{R} \rightarrow \mathbb{R}^{2 m}$ with compact support:

$$
\left\langle\left\langle w_{1}, w_{2}\right\rangle\right\rangle:=\int_{-\infty}^{\infty} w_{1}^{T}(t) Q_{e} w_{2}(t) d t .
$$

This is again a nondegenerate form. A subspace $\mathcal{D}$ of the space of locally integrable functions $w: \mathbb{R} \rightarrow \mathcal{U} \times \mathcal{U}^{*}$ of compact support is called a Dirac structure if $\mathcal{D}=$ $\mathcal{D}^{\Perp}$, where $\Perp$ denotes the orthogonal complement with respect to the form $\langle\langle\cdot, \cdot\rangle\rangle$. Equivalently, $D$ is a Dirac structure if $\langle\langle\cdot, \cdot\rangle\rangle$ is zero restricted to $\mathcal{D}$, and moreover $\mathcal{D}$ is maximal with respect to this property. We arrive at the following behavioral characterization of a conservative linear port-Hamiltonian system explored in [24], based on the concept of a state space port-Hamiltonian system originating in $[23,12$, 7].

Definition 4.6. A behavior $\mathcal{B}$ defines a conservative port-Hamiltonian system if $\mathcal{B}$ is a Dirac structure with respect to the form $\langle\langle\cdot, \cdot\rangle\rangle$ on the space $\mathcal{C}\left(\mathbb{R}, \mathbb{R}^{2 m}\right)$.

Hence the system $w=M\left(\frac{d}{d t}\right) \ell$ is conservative port-Hamiltonian if

$$
\int_{-\infty}^{\infty} w_{1}^{T}(t) Q_{e} w_{2}(t) d t=\int_{-\infty}^{\infty}\left(M\left(\frac{d}{d t}\right) \ell_{1}(t)\right)^{T} Q_{e} M\left(\frac{d}{d t}\right) \ell_{2}(t)=0
$$

for all $\ell_{1}, \ell_{2}$ with compact support, and furthermore the system is maximal with regard to this property. It follows that $w=M\left(\frac{d}{d t}\right) \ell$ is conservative port-Hamiltonian if and only if for all $s \in \mathbb{C}$

$$
M^{T}(-s) Q_{e} M(s)=0, \quad \operatorname{dim} M(s)=m\left(=\frac{1}{2} \operatorname{dim} \mathcal{W}\right) .
$$

As before, this is equivalent to the existence of a two-variable polynomial matrix $\Psi(\zeta, \eta)$ such that

$$
M^{T}(\zeta) Q_{e} M(\eta)=(\zeta+\eta) \Psi(\zeta, \eta)
$$

Since $\Psi(\zeta, \eta)=\Psi^{T}(\eta, \zeta)$, it follows that the coefficient matrix $\tilde{\Psi}$ is symmetric, and consequently $\Psi(\zeta, \eta)$ can be minimally factorized as

$$
\Psi(\zeta, \eta)=Z^{T}(\zeta) Q_{i} Z(\eta), \quad Q_{i}=Q_{i}^{T},
$$

for some $n \times 2 m$ polynomial matrix $Z(\xi)$ and some symmetric $n \times n$ full-rank matrix $Q_{i}$. We obtain the following theorem which parallels Theorem 4.2.

THEOREM 4.7. Let $w=M\left(\frac{d}{d t}\right) \ell$ with behavior $\mathcal{B}$ be a conservative port-Hamiltonian system. Consider the minimal factorization (4.20). Then $x=Z\left(\frac{d}{d t}\right) \ell$ is a minimal state map as in section 2, with McMillan degree equal to $n$. Furthermore, for every $w_{i}=M\left(\frac{d}{d t}\right) \ell_{i} \in \mathcal{B}$ with associated state trajectories $x_{i}:=Z\left(\frac{d}{d t}\right) \ell_{i}, i=1,2$, it holds that

$$
\frac{d}{d t} x_{1}^{T} Q_{i} x_{2}=w_{1}^{T} Q_{e} w_{2}
$$

Proof. The proof is completely analogous to the proof of Theorem 4.2. Consider the equality $-M(-\zeta)+M(\eta)=(\zeta+\eta) \Pi_{M}(\zeta, \eta)$. Substitution of $M(\eta)=M(-\zeta)+$ $(\zeta+\eta) \Pi_{M}(\zeta, \eta)$ in (4.19) leads to

$$
M^{T}(\zeta) Q_{e} \Pi_{M}(\zeta, \eta)=\Psi(\zeta, \eta) .
$$

Copyright $@$ by SIAM. Unauthorized reproduction of this article is prohibited. 
Substitution of (4.20) and a minimal factorization $\Pi_{M}(\zeta, \eta)=Y_{M}^{T}(\zeta) X_{M}(\eta)$ in (4.22) then yields

$$
M^{T}(\zeta) Q_{e} Y_{M}^{T}(\zeta) X_{M}(\eta)=Z^{T}(\zeta) Q_{i} Z(\eta) .
$$

Hence, up to premultiplication by an invertible matrix, $X_{M}(\eta)=Z(\eta)$, and thus $x=Z\left(\frac{d}{d t}\right) \ell$ is a minimal state map for the full behavior $\mathcal{B}_{\text {full }}$. Finally, combine (4.19) and (4.20) to conclude that

$$
M^{T}(\zeta) Q_{e} M(\eta)=(\zeta+\eta) \Psi(\zeta, \eta)=(\zeta+\eta) Z^{T}(\zeta) Q_{i} Z(\eta),
$$

which implies that for every $w_{i}=M\left(\frac{d}{d t}\right) \ell_{i} \in \mathcal{B}=\mathcal{B}_{\text {ext }}$ and $x_{i}=Z\left(\frac{d}{d t}\right) \ell_{i}, i=1,2$, the equality (4.21) holds.

Remark 4.8. The quadratic form $\frac{1}{2} x^{T} Q_{i} x$ defines the internal energy of the system, while $\frac{1}{2} w^{T} Q_{e} w$ corresponds to the supply rate of the system. In fact, for $w=\left[\begin{array}{l}y \\ u\end{array}\right]$ we have $\frac{1}{2} w^{T} Q_{e} w=\frac{1}{2}\left(y^{T} u+u^{T} y\right)=u^{T} y$, which is the standard passivity supply rate. Thus (4.21) amounts to the conservation of energy

$$
\frac{d}{d t} \frac{1}{2} x^{T} Q_{i} x=u^{T} y
$$

which is the usual definition of a lossless system in case $Q_{i}>0$. In fact, an inputoutput system given in transfer matrix format $y(s)=G(s) u(s), u \in \mathcal{U}, y \in \mathcal{U}^{*}$, is conservative port-Hamiltonian if and only if

$$
G(s)=-G^{T}(-s) .
$$

This is the starting point taken in $[23,12]$, where the existence of the internal symmetric form $Q_{i}$ is derived from the state space isomorphism theorem. In the current treatment, however, $Q_{i}$ is explicitly constructed using (4.20). Among others, this has the advantage that the signature of $Q_{i}$ can be directly determined; in particular it can be immediately verified if $Q_{i}$ is positive-definite.

4.3. Time-reversible systems. In this subsection we investigate the structure of state maps and the resulting state spaces for time-reversible systems. Let $V_{e}$ : $\mathbb{R}^{q} \rightarrow \mathbb{R}^{q}$ be a linear involution, i.e., $V_{e}^{2}=I_{q}$. Define the time-reversal operator $\mathcal{R}: \mathcal{L}_{1}^{\text {loc }}\left(\mathbb{R}, \mathbb{R}^{q}\right) \rightarrow \mathcal{L}_{1}^{\text {loc }}\left(\mathbb{R}, \mathbb{R}^{q}\right)$ corresponding to $V_{e}$ as

$$
(\mathcal{R} w)(t):=V_{e} w(-t), \quad t \in \mathbb{R},
$$

for any $w \in \mathcal{L}_{1}^{\text {loc }}\left(\mathbb{R}, \mathbb{R}^{q}\right)$. Then a behavior $\mathcal{B}$ is called time-reversible if it is invariant under $\mathcal{R}$, that is,

$$
\mathcal{R}(\mathcal{B})=\mathcal{B}
$$

The following result was obtained in [8].

Proposition 4.9. Every time-reversible behavior $\mathcal{B}$ described by a set of higherorder linear differential equations can be represented as $R\left(\frac{d}{d t}\right) w=0$ with $R(\xi)$ satisfying

$$
R(-\xi) V_{e}=S R(\xi)
$$

for some signature matrix $S$, that is, a diagonal matrix

$$
S=\left[\begin{array}{cc}
I_{p_{1}} & 0 \\
0 & -I_{p_{2}}
\end{array}\right]
$$

Copyright $@$ by SIAM. Unauthorized reproduction of this article is prohibited. 
with $p_{1}+p_{2}=p$. Conversely, if $R(\xi)$ satisfies (4.28), then the system $R\left(\frac{d}{d t}\right) w=0$ is time-reversible.

Our main result is that the property (4.28) translates into the following property of state maps obtained from factorization of $\Pi(\zeta, \eta)$.

Proposition 4.10. Consider a time-reversible system $R\left(\frac{d}{d t}\right) w=0$ with $R(\xi)$ satisfying (4.28). Consider any state map $x=X\left(\frac{d}{d t}\right) w$, with $x \in \mathbb{R}^{n}$, obtained from a minimal factorization $\Pi(\zeta, \eta)=Y^{T}(\zeta) X(\eta)$, where as before $R(-\zeta)-R(\eta)=(\zeta+$ $\eta) \Pi(\zeta, \eta)$. Then there exists a nonsingular map $V_{i}: \mathbb{R}^{n} \rightarrow \mathbb{R}^{n}$ which is again an involution, that is, $V_{i}^{2}=I_{n}$, such that

$$
X(\xi) V_{e}=V_{i} X(-\xi) .
$$

Proof. By (4.28),

$$
(R(-\zeta)-R(\eta)) V_{e}=S(R(\zeta)-R(-\eta))
$$

and hence

$$
(\zeta+\eta) \Pi(\zeta, \eta) V_{e}=-(\zeta+\eta) S \Pi(-\zeta,-\eta)
$$

or, equivalently,

$$
\Pi(\zeta, \eta) V_{e}=-S \Pi(-\zeta,-\eta)
$$

Thus for a minimal factorization $\Pi(\zeta, \eta)=Y^{T}(\zeta) X(\eta)$ we obtain from Proposition 2.4 that there exists a unique $V_{i}$ such that $X(\xi) V_{e}=V_{i} X(-\xi)$. This furthermore implies

$$
X(\xi)=X(\xi) V_{e} V_{e}=V_{i} X(-\xi) V_{e}=V_{i} V_{i} X(\xi),
$$

and hence $V_{i}^{2}=I_{n}$.

The same result for minimal state space realizations of a transfer matrix $G(s)$ satisfying the time-reversibility condition $G(s)=G(-s)$ (corresponding to $V_{e}=I$ ) was already obtained, using the state-space isomorphism theorem, in [26]. Various extensions can be found in [8]. The contribution of the above proposition is that the existence of the internal involution $V_{i}$ is directly inferred from the properties of the (not necessarily minimal!) state map $x=X\left(\frac{d}{d t}\right) w$ obtained from a minimal factorization of $\Pi(\zeta, \eta)$.

5. Conclusions. In this paper we have developed a novel approach to the construction of state variables for systems described by sets of higher-order linear differential equations. By starting from the characterization of state as an equivalence relation among solutions, state maps are directly constructed by repeated "integration by parts," using the calculus of bilinear differential forms and factorization of two-variable polynomial matrices, also relating to the adjoint system. We have applied our approach to Hamiltonian and time-reversible systems, yielding canonical structures on their state space in a constructive way. The basic idea underlying the proposed construction, that is, "integration by parts" and Lagrange's identity, seems sufficiently general to be extendable to other system classes, such as time-varying linear systems and systems of linear PDEs, as well as nonlinear systems. More specific research questions include the construction of state maps for uncontrollable Hamiltonian systems, making use of their behavioral characterization obtained in [16], as well 
as the characterization of the set of storage functions of (uncontrollable) dissipative systems, continuing the work of [27]. Furthermore, in a future paper we will apply the state map construction to state space realization theory, building upon the results obtained in section 2.5.

\section{REFERENCES}

[1] B. D. O. Anderson And E. I. Jury, Generalized Bezoutian and Sylvester matrices in linear multivariable linear control, IEEE Trans. Automat. Control, 21 (1976), pp. 551-556.

[2] R. W. Brockett, Control theory and analytical mechanics, in The 1976 Ames Research Center (NASA) Conference on the Geometric Theory of Nonlinear Waves (Moffett Field, CA, June 16-23, 1976), Lie Groups: History, Frontiers and Appl. VII, R. Hermann, ed., Math. Sci. Press, Brookline, MA, 1977, pp. 1-48.

[3] R. W. Brockett, Path integrals, Lyapunov functions, and quadratic minimization, in Proceedings of the 4th Allerton Conference on Circuit and System Theory, University of Illinois, Monticello, IL, 1966, pp. 685-698.

4] R. W. Brockett, Finite Dimensional Linear Systems, John Wiley \& Sons, New York, 1970.

[5] R. W. Brockett and A. Rahimi, Lie algebras and linear differential equations, in Ordinary Differential Equations, L. Weiss, ed., Academic Press, New York, 1972, pp. 379-386.

[6] P. E. Crouch and A. J. Van der Schaft, Variational and Hamiltonian Control Systems, Springer-Verlag, Berlin, 1987.

[7] M. DALSMO AND A. VAN DER SChAFt, On representations and integrability of mathematical structures in energy-conserving physical systems, SIAM J. Control Optim., 37 (1998), pp. 54-91.

[8] F. Fagnani and J. C. Willems, Representations of time-reversible systems, J. Math. Systems Estim. Control, 1 (1991), pp. 5-28.

[9] P. A. Fuhrmann, P. Rapisarda, and Y. Yamamoto, On the state of behaviors, Linear Algebra Appl., 424 (2007), pp. 570-614.

[10] E. L. Ince, Ordinary Differential Equations, Dover Publications, New York, 1956. (First edition published in 1926 by Longmans, Green and Co., London.)

[11] T. Kailath, Linear Systems, Prentice-Hall, Englewood Cliffs, NJ, 1980.

[12] B. Maschke And A. J. VAn Der Schaft, Port-controlled Hamiltonian systems: Modelling origins and system theoretic properties, in Proceedings of the 2nd IFAC Symposium on Nonlinear Control Systems Design (NOLCOS 92), Bordeaux, France, M. Fliess, ed., Pergamon Press, Oxford, UK, 1992, pp. 282-288.

[13] J. W. Nieuwenhuis and J. C. Willems, Duality for linear time invariant finite dimensional systems, in Analysis and Optimization of Systems, A. Bensoussan and J. L. Lions, eds., Lecture Notes in Control and Inform. Sci. 111, Springer, Berlin, 1988, pp. 13-21.

[14] J. W. Polderman and J. C. Willems, Introduction to Mathematical System Theory: A Behavioral Approach, Springer-Verlag, Berlin, 1997.

[15] P. Rapisarda And A. J. VAn DeR Schaft, State maps from bilinear differential forms, in Proceedings of the 19th International Symposium on Mathematical Theory of Networks and Systems (MTNS, July 5-9, 2010), MTA SZTAKI (Computer and Automation Research Institute, Hungarian Academy of Sciences), Budapest, Hungary, 2010, pp. 685-689.

[16] P. Rapisarda and H. L. Trentelman, Linear Hamiltonian behaviors and bilinear differential forms, SIAM J. Control Optim., 43 (2004), pp. 769-791.

[17] P. Rapisarda and J. C. Willems, State maps for linear systems, SIAM J. Control Optim., 35 (1997), pp. 1053-1091.

[18] A. J. van der Schaft, Time-reversible Hamiltonian systems, Systems Control Lett., 1 (1982), pp. 295-300.

[19] A. J. VAn Der Schaft, System Theoretic Description of Physical Systems, CWI Tract 3, Stichting Mathematisch Centrum, Centrum voor Wiskunde en Informatica, Amsterdam, 1984.

[20] A. J. VAn DeR Schaft, System theory and mechanics, in Three Decades of Mathematical System Theory, H. Nijmeijer and J. M. Schumacher, eds., Lecture Notes in Control and Inform. Sci. 135, Springer, Berlin, 1989, pp. 426-452.

[21] A. J. VAN DER SCHAFT, Duality for linear systems: External and state space characterization of the adjoint system, in Analysis of Controlled Dynamical Systems, B. Bonnard, B. Bride, J. P. Gauthier, and I. Kupka, eds., Birkhaüser Boston, Boston, MA, 1991, pp. 393-403. 
[22] A. J. VAN DER SCHAFT, Representing a nonlinear input-output differential equation as an inputstate-output system, in Open Problems in Mathematical Systems Control and Theory, V. Blondel, E. D. Sontag, M. Vidyasagar, and J. C.Willems, eds., Comm. Control Engrg. Ser., Springer, London, 1999, pp. 171-176.

[23] A. J. van Der Schaft And B. M. MaschKe, The Hamiltonian formulation of energy conserving physical systems with external ports, Archiv für Elektronik und Übertragungstechnik, 49 (1995), pp. 362-371.

[24] A. J. van DER SChaft AND R. PolyUga, Structure-preserving model reduction of complex physical systems, in Proceedings of the 48th IEEE Conference on Decision and Control, Shanghai, China, 2009, pp. 4322-4327.

[25] A. J. VAn Der Schaft And P. RAPISARda, From external to internal system decompositions, in Proceedings of the 18th IFAC World Congress, Milan, Italy, 2011, pp. 3316-3320.

[26] J. C. Willems, Time reversibility in deterministic and stochastic dynamical systems, in Recent Developments in Variable Structure Systems, Economics and Biology, R. R. Mohler and A. Ruberti, eds., Lecture Notes in Econom. and Math. Systems 162, Springer, Berlin, New York, 1978, pp. 318-326.

[27] J. C. Willems and H. L. Trentelman, On quadratic differential forms, SIAM J. Control Optim., 36 (1998), pp. 1703-1749.

Copyright (c) by SIAM. Unauthorized reproduction of this article is prohibited. 